Pontifícia Universidade C Católica DO RIO DE JANEIRO

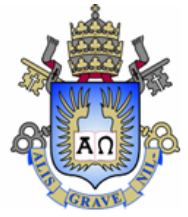

William Marques Manhães

\title{
Lagrangians for Electromechanical Systems
}

Graduation Project presented to the Department of Mechanical Engineering at PUC-Rio

Project Supervisor: Rubens Sampaio Filho

Rio de Janeiro

July, 2019 


\section{ACKNOWLEDGMENTS}

Thanks to Rubens Sampaio and Roberta Lima for the opportunity.

I would like to thank some friends: Fernanda, Carol, Luís, Mateus and Roberta

Thanks to my beloved family for making this possible.

Special thanks to Gabi who told me people should do what they love. 


\section{ABSTRACT}

\section{Lagrangians for Electromechanical Systems}

Electromechanical systems are very common. The importance of constructing the dynamical equations of motors coupled with mechanical subsystems suggests a new strategy . In the literature, often, the derivation of the dynamical equations is wrong. One thinks that the standard derivations of the dynamical equations of purely mechanical systems can be mimicked to electromechanical systems. Unfortunately, it cannot. The main reason is that in electromechanical systems one deals with the presence of electromagnetic fields, continuous entities. These fields store electrical and mechanical energies. In purely mechanical systems the conservative mechanical energy is stored as elastic or gravitational energy, and the nonconservative terms enter the equation as nonconservative forces. This cannot be done in electromechanical systems. This project shows the right way to derive the dynamical equations applying the results for systems formed by a motor, a coupling mechanism, and a mechanical subsystem.

Keywords : Electromechanical Systems. Coupled Systems. Lagrangians 


\section{RESUMO}

\section{Formulação Lagrangiana para Sistemas Eletromecânicos}

Sistemas eletromecânicos são muito comuns. A importância de construir as equações dinâmicas de motores acoplados a subsistemas mecânicos sugere uma nova estratégia. $\mathrm{Na}$ literatura, muitas vezes, a derivação das equações da dinâmica está errada. Pensa-se que as derivações padrão da dinâmica de sistemas puramente mecânicos podem ser imitadas para sistemas eletromecânicos. Infelizmente, isso não é possível. A principal razão é que em sistemas eletromecânicos se lida com a presença de campos eletromagnéticos, entidades contínuas. Esses campos armazenam energias eletromagnéticas e mecânicas. Em sistemas puramente mecânicos, a energia mecânica conservadora é armazenada como energia elástica ou gravitacional, e os termos não conservativos entram na equação como forças não conservativas. Isso não pode ser feito em sistemas eletromecânicos. Este projeto mostra o caminho certo para derivar as equações da dinâmica, aplicando os resultados para sistemas formados por um motor, um mecanismo de acoplamento e um subsistema mecânico.

Palavras-chave : Sistemas eletromecânicos. Sistemas acoplados. Formulação Lagrangiana 


\section{Contents}

1 Introduction $\quad 6$

2 Legendre Transformations $\quad 7$

2.1 In Thermodynamics . . . . . . . . . . . . . . . . . . . . . . . 8

2.2 In Classical Mechanics . . . . . . . . . . . . . . . . . . . . . . 9

3 Energy Considerations $\quad 10$

3.1 Energy Balance Relationships . . . . . . . . . . . . . . . . . . 10

3.2 Energy and Coenergy Relations . . . . . . . . . . . . . . . . . 15

4 Modeling Technique for Electromechanical Systems $\quad 17$

4.1 Electromechanical Coupling . . . . . . . . . . . . . . . . 17

4.2 Lagrangians for Electromechanical Systems . . . . . . . . . . . . . . . . 18

4.3 Step by Step: Lagrangians for Electromechanical Systems . . . . . . . . . . 19

5 Dynamics of Electromechanical Systems 22

5.1 Scotch-Yoke Mechanism . . . . . . . . . . . . . . . . . 22

5.2 Crank-Slider Mechanism . . . . . . . . . . . . . . . . . 26

6 Conclusions $\quad 30$

$\begin{array}{lll}7 & \text { References } & 30\end{array}$ 


\section{List of Figures}

1 (a) A curve in the XY plane (b) B is the value where the line crosses the $\mathrm{Y}$ axis and $\mathrm{S}$ is the slope of the line (c) Notice that the curve can be represented by a family of lines. Every curve has a pair $(\mathrm{Y}, \mathrm{X})$ and every

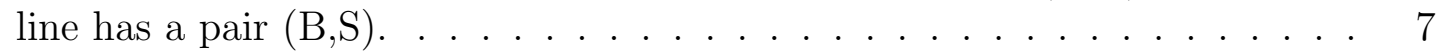

2 Thermodynamic square. . . . . . . . . . . . . . . . 8

3 Legendre Transformations in Classical Mechanics. Image taken from [13]. . 9

4 Energy transfer in an electromechanical system. . . . . . . . . . . . . . . . 10

5 (a) Electromechanical system with a magnetic coupling field (b) Electromechanical system with an electrical coupling field . . . . . . . . . . . . 12

6 Graphics of energy and coenergy for linear and non-linear systems . . . . . 16

7 Cart-motor system with a scotch-yoke mechanism. . . . . . . . . . . . . 22

8 DC motor. . . . . . . . . . . . . . . . . . . . . . 22

9 Electromechanical system with a crank-slider mechanism. . . . . . . . 26

10 DC motor. . . . . . . . . . . . . . . . . . . . 26 


\section{Introduction}

Modeling plays a necessary role in the design of dynamical systems. It helps to describe the behavior of a system and to acquire a better understanding of its functionality. In the engineering domain, physical systems such as mechanical, electrical, fluid and thermo can be explained by energy in its most diverse forms. Energy is always a common name when dealing with a specifique nature. While studying systems composed by two different parts, energy does not seem to be enough. It needs a complement. The complementary energy - coenergy - has an important role in systems with mutual interactions. These systems share an unique characteristic: Coupling terms. Two subsystems are only together if coupled. For that reason, energy alone is not sufficient. The coenergy helps to identify terms of mutual interaction. If only energy is discussed, as it is done when dealing with one system alone with no subsystems, it turns out to be impossible to identify coupling terms. The difference between energy and coenergy must be presented. It is noticiable that the pair energy-coenergy works as an universal language between physical systems. With one it is easy to acquire the other through a variable modification. The Legendre transformation links several energy functions in the physical domain. One of them is the energy-coenergy transformation. Useful but not always possible. Another interesting transformation enters the field of Thermodynamics, it relates the Internal Energy with Helmholtz Free Energy, Enthalpy and Gibbs Free Energy. Other formulations such as Lagrangians, Colagrangians, Hamiltonians and Cohamiltonians are also functions related through a Legendre Transformation.

Couplings are not always connecting two different subsystems. It is possible to have coupling between two parts of the same origin. For example, some electromechanical systems examples in this project have both: An electromechanical and a mechanical coupling. Electromechanical systems are characterized by a mutual influence between mechanical and electrical parts [10,9]. These parts share an electromechanical coupling, necessarily. For the section of DC motors coupled to mechanical parts, two systems are discussed. They have the same electromechanical coupling. Their difference lies in the choice of the mechanical coupling mechanism. The first objective is to formulate a Lagragian capable of describing any electromechanical system. The mechanical coupling of the first system is made by a mechanism called slider-crank, see [3, 1]. The second uses a mechanism called scotch-yoke, see $[14,16]$. These systems have an interesting feature: they work as a sort of master-slave condition. The motor rotates and the mechanical part reacts. 


\section{Legendre Transformations}

The Legendre transformation is used to transitate between functions. It consists in the change of one or more independent variables of a particular function, constructing a new one. The new function is a new form of interpreting the old one. As an example, take the fundamental relation $Y(X)$. Notice this relation is a function of only one independent variable $X$. Geometrically, it is represented by a curve in a plane with coordinates $Y$ and $X$. The derivative of the function $Y$ in respect to $X$ is the slope of the curve. Now, the slope $S$ is considered also as an independent variable, one can easily write $Y(S)$. Now, $Y(X)$ and $Y(S)$ represent the same curve. The objective is to demonstrate that a curve can also be represented equally by a family of tangent lines. Every line is described by two numbers $S$ and $B$, where $B$ is the value where the line crosses the $Y$ axis. When $Y(X)$ describes a curve with a family of points, $B(S)$ describes the same curve with a family of lines.

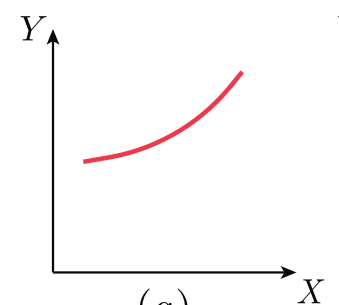

(a)

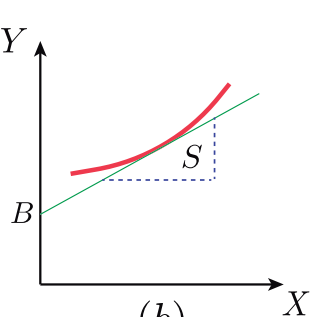

(b)

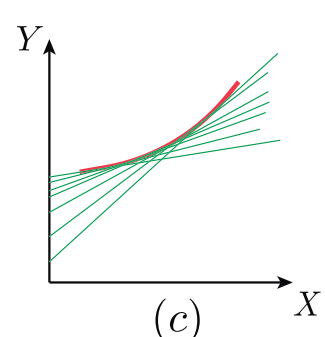

(c)

Figure 1: (a) A curve in the XY plane (b) B is the value where the line crosses the $\mathrm{Y}$ axis and $\mathrm{S}$ is the slope of the line (c) Notice that the curve can be represented by a family of lines. Every curve has a pair $(\mathrm{Y}, \mathrm{X})$ and every line has a pair $(\mathrm{B}, \mathrm{S})$.

Take as an example a curve $Y$, which is a function of only one independent variable $X$. The curve $Y$ used in the following example is different from the one represented in Figure 1 but both examples follow the same mechanism. Figure 1 is an important graphical aproach of what is actually happening to any curve submitted to a Legendre transformation. Take the derivation of this $Y$ curve with respect to its variable to obtain the slope $S$ of the curve. Through a relation caractherized by the division of the difference between two points of a line, basically a line equation, it is possible to correlate $S, Y, X$ and $B$ (which is the value where the line crosses the $Y$-axis).

$$
\begin{aligned}
& Y(X) \quad \rightarrow \quad S(X)=\frac{d Y}{d X} \rightarrow B(S), \\
& Y(X)=\frac{1}{4} X^{2} \quad, \quad S(X)=\frac{d Y}{d X}=\frac{1}{2} X .
\end{aligned}
$$

Through the equation of the line, it is possible to define $B(S)$ once $Y$ and $X$ are also functions of $S$ :

$$
X(S)=2 S \quad \rightarrow \quad \text { substituting in } Y(X) \quad \rightarrow \quad Y(S)=S^{2}
$$

equation of the line: $S=\frac{Y-B}{X-0} \quad$ or $\quad Y=B+S X \quad \rightarrow \quad B(S)=-S^{2}$. 
It is now evident that representing a curve with the function $Y(X)$ is not the only possibility. If necessary there are other ways to represent it. If the pair of points $(X, Y)$ is not an important information in the representation, the same curve can be represented by the pair $(S, B)$ which gives the slope of the curve and the value the line crosses the vertical axis. It is interesting to analyse graphically the equation $S=\frac{1}{2} X$ with the slope $S$ in the vertical axis and $X$ in the horizontal axis. The relation between $S$ and $X$ is linear. The areas beneath and above the line are $Y(S)$ and $Y(X)$, respectively. The areas are equal, which means that both representations are the same quantity, the same curve in different formalisms.

Each of the following alternative formulations presented throughout this section is appropriate for specific problems. The reason independent variables are changed is a matter of suitability. Some problems tend to be extremely complicated and even impossible in wrong formalisms.

\section{$2.1 \quad$ In Thermodynamics}

The art of Thermodynamics lies in the selection of the particular formulation, [7]. In this section four of the most common thermodynamic potetials related through a Legendre transformation are presented. The Helmholtz Free Energy $F(T, V)$, which has natural variables temperature $T$ and volume $V$, is one of the partial Legendre transformations of the Internal Energy $U(S, V)$, which depends on the variables entropy $S$ and also the volume $V$. The relationship between these two functions is made by changing one or two variables. The Legendre transformation of $U(S, V)$ to $F(T, V)$ is characterized by the change of the entropy $S$ by the temperature $T$. If the volume $V$ of $U(S, V)$ is changed by the pressure $P$, the Internal Energy $U(S, V)$ is transformed to Enthalpy $H(S, P)$, it is another example of Legendre transformation. Finally, if both variables are changed, $S$ by $T$ and $V$ by $P$, another potential called the Gibbs Free Energy $G(T, P)$ is created. All of the functions $F, H$ and $G$ are Internal Energies $U$ in other formalism. A representation resuming all of the transformations is called the thermodynamic square.

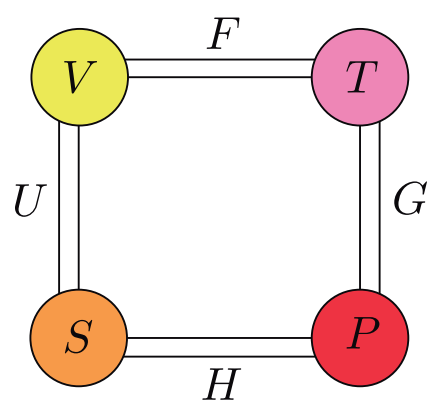

Figure 2: Thermodynamic square. 


\subsection{In Classical Mechanics}

In Classical Mechanics, there are several formulations. Some are very famous, as the Newtonian, Lagrangian and Hamiltonian formulations. Others are not frequently used, as the Colagrangian and the Cohamiltonian. From the Newtonian, it is easy to formulate the Lagrangian. They are not connected through a Legendre Transform, but the equations of Newton and Lagrange are basically the same. With an exception of the Newtonian, all other formulations present an energy approach. By changing independent variables and modifing energy functions, Lagrangians, Colagrangians, Hamiltonians and Cohamiltonians are also functions related through a Legendre Transformation.

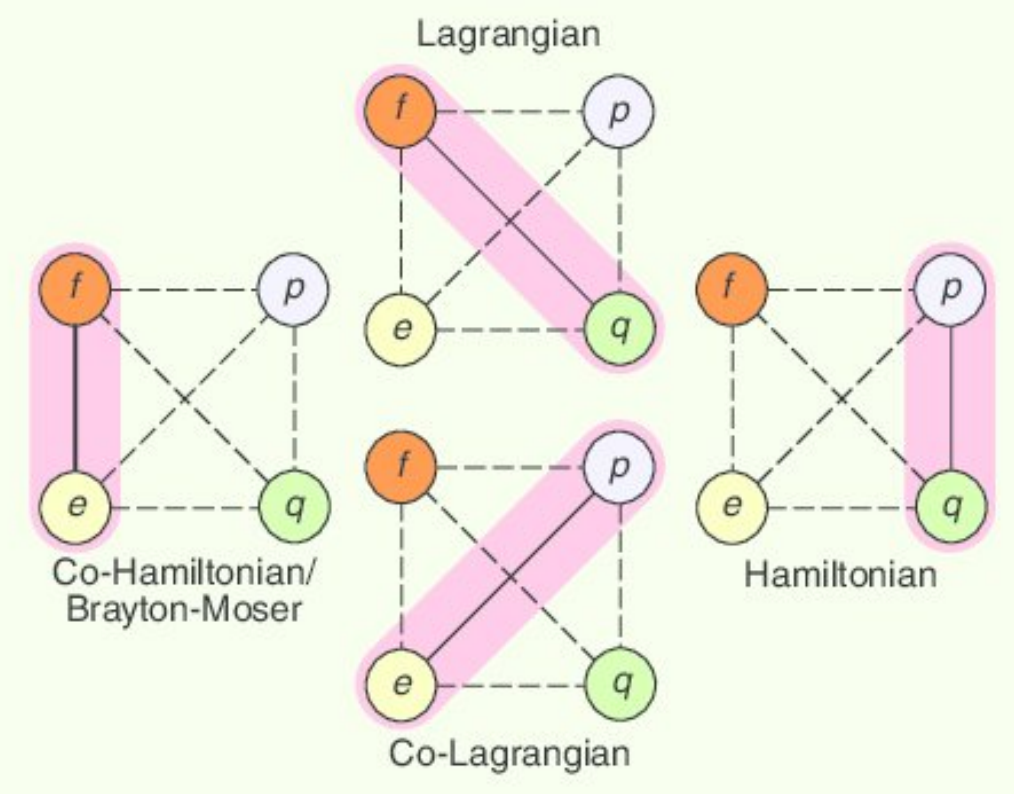

Figure 3: Legendre Transformations in Classical Mechanics. Image taken from [13].

Taking $\mathrm{f}, \mathrm{q}, \mathrm{p}$ and $\mathrm{q}$ as independent variables:

From the Lagrangian to the Hamiltonian:

$$
L(f, q) \Rightarrow H(p, q)
$$

From the Lagrangian to the Cohamiltonian:

$$
L(f, q) \Rightarrow C O H(f, e) .
$$

From the Lagrangian to the Colagrangian:

$$
L(f, q) \Rightarrow C O L(p, e)
$$




\section{$3 \quad$ Energy Considerations}

\subsection{Energy Balance Relationships}

In this project, for a better understanding, electromechanical systems are considered as lumped-parameter systems. They are described by a finite number of mechanical and electrical variables. In these systems, interaction occurs through eletrostatic and eletromagnetic fields. These fields are called coupling fields. They are common to electrical and mechanical parts. Both subsystems will always interact and the interaction causes energy transfer.

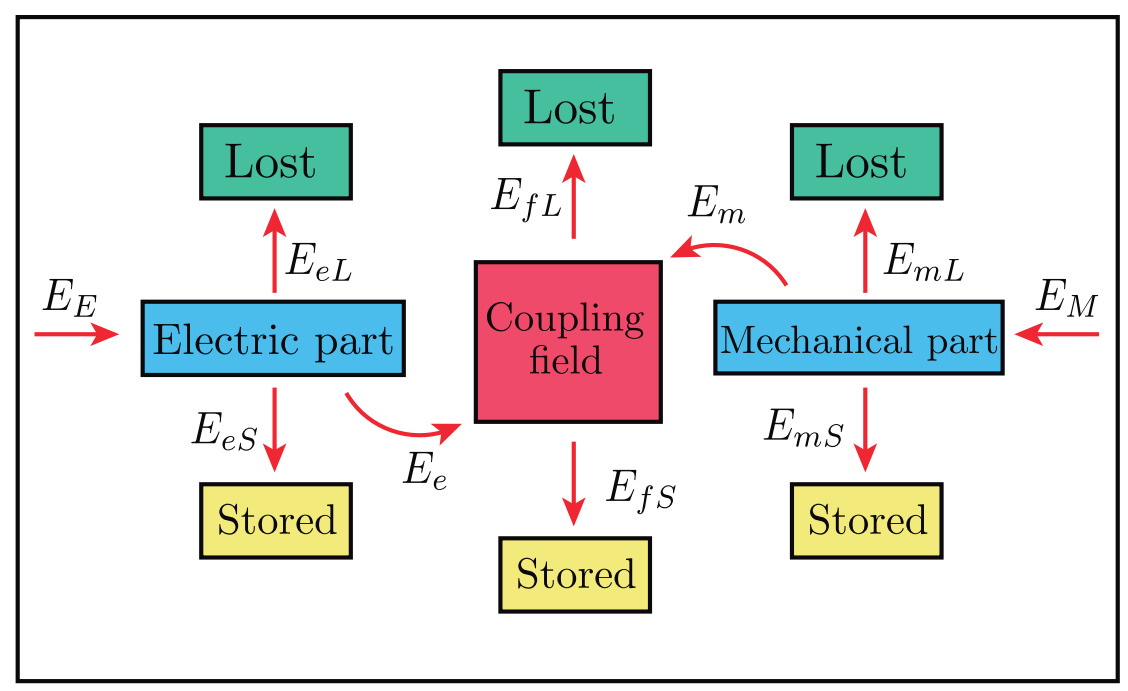

Figure 4: Energy transfer in an electromechanical system.

A simplified form of an electromechanical system can be represented by Figure 4. It shows how energy transfers from one point to another. Now it is apparent how electrical and mechanical parts interact in a system. Electromechanical systems are coupled systems by nature. They interact through a coupling field. $E_{m}$ is the energy transferred to the coupling field by the mechanical subsystem, $E_{m L}$ the loss also associated to the mechanical part and $E_{m S}$ is the energy stored in the mechanical elements. It is possible to describe the total energy supplied by the mechanical part as $E_{M}$. The same can be done for the electrical part. $E_{e}$ is the energy transferred to the coupling field by the electrical part, $E_{e L}$ is the loss also associated to the electric subsystem and $E_{e S}$ is the energy stored in electric or magnetic fields which are not coupled to the mechanical subsystem. It is possible to describe the energy supplied by the electrical part as $E_{E}$. Analysing the directions of the arrows in figure 4 , the following equations are easily obtained. 
For the energy supplied to both parts, one has

$$
\begin{gathered}
E_{M}=E_{m}+E_{m L}+E_{m S}, \\
E_{E}=E_{e}+E_{e L}+E_{e S} .
\end{gathered}
$$

Now for the total energy transferred to the coupling field we must separate the energy stored from the energy dissipated. For $E_{f S}$ being the total energy stored and $E_{f L}$ the total energy dissipated in the coupling field, it is possible to define the total energy transferred to the coupling field as:

$$
E_{F}=E_{e}+E_{m}=E_{f S}+E_{f L}
$$

To demonstrate the equality of the equation 10 we define $E_{f S}$ and $E_{f L}$. Both are the difference between the total energy supplied and the sum of the energy stored with the energy lost, for each subsystem :

$$
\begin{gathered}
E_{f S}=E_{e}-\left(E_{e L}+E_{e S}\right), \\
E_{f L}=E_{m}-\left(E_{m L}+E_{m S}\right),
\end{gathered}
$$

Substituting 11 and 12 in 10 we have:

$$
E_{F}=\left(E_{e}-E_{e L}-E_{e S}\right)+\left(E_{m}-E_{m L}-E_{m S}\right),
$$

Substituting equations 8 and 9 in 13 gives,

$$
E_{F}=E_{e}+E_{m}
$$

The total energy transferred to the coupling field is independent of the energy dissipated in the mechanical or electrical part. It is also independent of the energy stored outside the coupling terminals. Thus, if the losses of the coupling field are not taken into account, the field between the coupling terminals is said to be conservative. It can be compared to a box that contains only an electric or a magnetic field. The value and the energy storaged inside is affected by electrical and mechanical variables. The energy put into the system by the mechanical and electrical pairs is stored in the field and can be recovered completely. That is the reason an assumption of a conservative coupling field is valid. Consider two examples of electromechanical systems. One has a magnetic field storage between coupling terminals and the other an electric field storage. Both fields interact with the mechanical subsystem by moving a plate. The electric subsystem is composed by a voltage $v$, a current $\dot{q}$ representing the time derivative of the electric charge $q$, an inductance $l$, a resistance $r$ and a coupling voltage denoted by $e_{f}$. The mechanical subsystem has a plate of mass $m$, a spring of stiffness $k$, a force $f$ externally applied, a damper with damping coefficient $b$ and a force of electric or magnetic origin $f_{e}$. In systems $a$ and $b, x$ is the displacement and $x_{0}$ is the equilibrium position of the plate. 


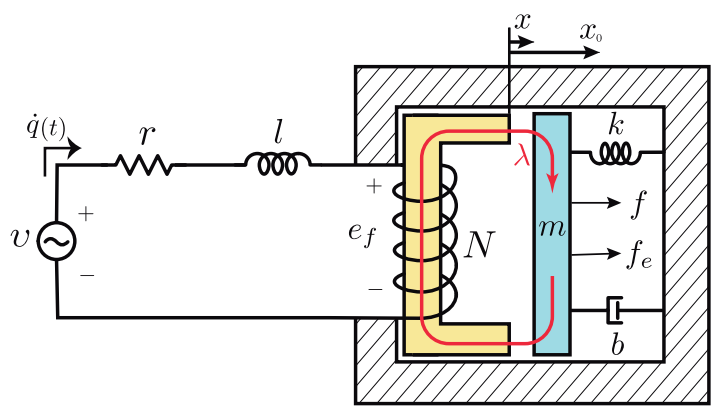

(a)

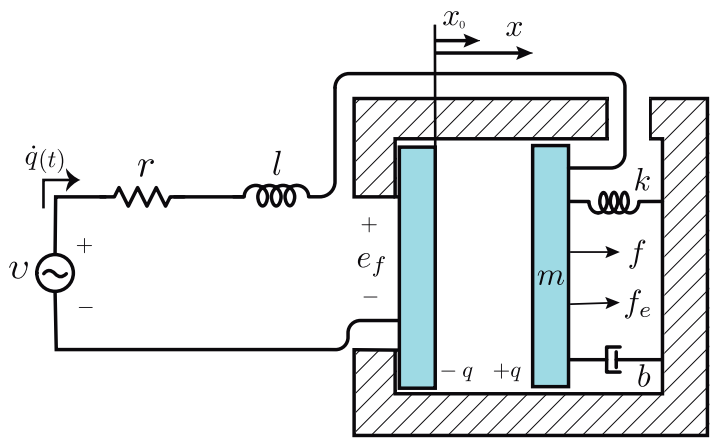

(b)

Figure 5: (a) Electromechanical system with a magnetic coupling field (b) Electromechanical system with an electrical coupling field

These two electromechanical systems are very simple. To obtain the equations of the dynamics in this case is trivial. Newton's and Kirchhoff's law applied to systems $(a)$ and (b) gives

$$
\begin{gathered}
m \ddot{x}+b \dot{x}+k\left(x-x_{0}\right)-f_{e}=f, \\
l \ddot{q}+r \dot{q}+e_{f}=v,
\end{gathered}
$$

After multiplying equation 16 by the current $\dot{q}$ and equation 15 by the velocity $\dot{x}$ we get two powers. Integrating both equations in time, we get two equalities of energy equations for

$$
\begin{gathered}
\int f \dot{x} d t=\int m \ddot{x} \dot{x} d t+\int b \dot{x}^{2} d t+\int k\left(x-x_{0}\right) \dot{x} d t-\int f_{e} \dot{x} d t \\
\qquad v \dot{q} d t=\int l \ddot{q} \dot{q} d t+\int r \dot{q}^{2} d t+\int e_{f} \dot{q} d t .
\end{gathered}
$$


Equations 17 and 18 also represent the energy supplied by the mechanical and the electrical part. Comparing equation 8 to equation 17 leads to

$$
\begin{gathered}
E_{M}=\int f \dot{x} d t \\
E_{m S}=\int m \ddot{x} \dot{x} d t+\int k\left(x-x_{0}\right) \dot{x} d t \\
E_{m L}=\int b \dot{x}^{2} d t \\
E_{m}=-\int f_{e} \dot{x} d t .
\end{gathered}
$$

Now comparing equation 9 to equation 18 we get

$$
\begin{aligned}
& E_{E}=\int v \dot{q} d t, \\
& E_{e S}=\int l \ddot{q} \dot{q} d t, \\
& E_{e L}=\int r \dot{q}^{2} d t, \\
& E_{e}=\int e_{f} \dot{q} d t .
\end{aligned}
$$

Substituting equations 22 and 23 in 14 gives

$$
E_{F}=\int e_{f} \dot{q} d t-\int f_{e} \dot{x} d t
$$

or in differential form:

$$
\frac{d}{d t}\left(E_{F}\right)=e_{f} \dot{q}-f_{e} \dot{x}
$$

The total energy transferred to the coupling field $E_{F}$ for systems $(a)$ and $(b)$ is denoted by equation 28 . The examples presented do not include all possibilities. Note that this equation is valid only for one mechanical and one electrical terminal. Aiming to expand the equation to other circunstances, it should not only increase the number of terminals but also include a torque. This mechanical torque, as the mechanical force, can have a magnetic or an electric origin. The rate of change in the angular displacement $\dot{\alpha}$ must multiply the torque $\tau_{e}$ in order to form a power and be included in equation 28. For $N$ electrical and $M$ mechanical terminals:

$$
\begin{gathered}
\frac{d}{d t}\left(E_{F}\right)=\sum_{i=1}^{N}\left(e_{f} \dot{q}\right)_{i}-\left(\sum_{i=1}^{M}\left(f_{e} \dot{x}\right)_{i}+\sum_{i=1}^{M}\left(\tau_{e} \dot{\alpha}\right)_{i}\right) \\
\frac{d}{d t}\left(E_{F}\right)=\sum_{i=1}^{N}\left(e_{f} \dot{q}\right)_{i}-\left(\sum_{i=1}^{M}\left(f_{e}\right)_{i} \frac{d x_{i}}{d t}+\sum_{i=1}^{M}\left(\tau_{e}\right)_{i} \frac{d \alpha_{i}}{d t}\right) .
\end{gathered}
$$


Energy generated can be stored, but power can not. Conserving power is a way to conserve the rate at which the energy is transmitted. Equation 30 shows that the rate of the energy transferred to the coupling field is conserved, see [19]. In the systems presented, the displacement $x$ represents the influence of the mechanical part in the coupling field. It could also be represented by an angular displacement $\alpha$. These coordinates $x$ and $\alpha$ are not related through a constitutive equation. It is true that, when there is a constraint, one can be written as a function of the other, but their relation is not always imposed. It is not necessary. Therefore, it is possible to have one of them $(x$ or $\alpha)$, or both, to represent the state of the mechanical part. Note that for the electrical part, the choice is not so trivial. The electric charge $q$ and the flux linkage $\lambda$ are often chosen as coordinates to represent an electrical system. Once the electric coordinates are related through constutive equations and as the derivative of the flux linkage $(d \lambda / d t)$ equals the voltage between the terminals of the couplig field $\left(e_{f}\right)$. The constitutive equations for both possibilities are

$$
\begin{aligned}
& q=c \frac{d \lambda}{d t}, \\
& \lambda=l \frac{d q}{d t} .
\end{aligned}
$$

Only one coordinate is necessary. The choice of the electrical variable must be unique for any number of coupling terminals. Usually for systems with magnetic energy storage as $(a)$, the choice of $\lambda$ seems to be motivated by how an increasing of the flux linkage leads to an increasing of the energy transmitted to the system. The same approach is made for systems like $(b)$ with an electric energy storage, the choice of $q$ is motivated by how incremental changes in the coupling field influences in the energy transmission. For now, this choice is suitable to understand the principles of the energy transfer. It is chosen $\lambda$ for the system with magnetic energy storage and $q$ for the system with electric energy storage. Later in this project, the choice of only one coordinate has to be made to formulate an extended Lagrangian. It is not convenient to work with two coordinates for a system while having a constraint, although working with two coordinates seems to be possible, see interesting formulation of [11]. For now, it is possible to write the equations for a magnetic and an electric energy storage represented by each of its chosen coordinates. Being $\mathcal{T}_{m}$ and $\mathcal{V}_{e}$ the energy stored in a magnetic and in an electric field, respectively.

For the energy transferred to a magnetic coupling field one has

$$
\frac{d}{d t}\left(\mathcal{T}_{m}\right)=\sum_{i=1}^{N}(\dot{q})_{i} \frac{d \lambda_{i}}{d t}-\left(\sum_{i=1}^{M}\left(f_{e}\right)_{i} \frac{d x_{i}}{d t}+\sum_{i=1}^{M}\left(\tau_{e}\right)_{i} \frac{d \alpha_{i}}{d t}\right)
$$

and for the energy transferred to an electric coupling field

$$
\frac{d}{d t}\left(\mathcal{V}_{e}\right)=\sum_{i=1}^{N}\left(e_{f}\right)_{i} \frac{d q_{i}}{d t}-\left(\sum_{i=1}^{M}\left(f_{e}\right)_{i} \frac{d x_{i}}{d t}+\sum_{i=1}^{M}\left(\tau_{e}\right)_{i} \frac{d \alpha_{i}}{d t}\right)
$$


The left side of the equations 33 and 34 shows a term that represents the time rate of increase in energy stored. In the right side, the difference between two other terms. The first is the power input at the electrical terminals and the second is the power input at the mechanical terminals. Second term has a negative sign because the force $f_{e}$ and the torque $\tau_{e}$ are defined as acting on the mechanical node.

\subsection{Energy and Coenergy Relations}

In the study of electromechanical systems, the definition of coenergy must be presented. Energy and coenergy complements one another [13]. Take an example in the mechanical domain: a particle with mass $m$ moving in the positive $x$ direction. This model represents a non-relativistic situation. We are able to write the kinetic energy and the kinetic coenergy of the particle with a constitutive law. This constitutive law will relate the momentum $p$ with the velocity $v$. By substituting the constitutive law in the energy equation, the coenergy is obtained:

$$
\begin{gathered}
\text { Energy: } \frac{p^{2}}{2 m}, \\
\text { Constitutive law: } p=m v, \\
\text { Coenergy: } \frac{m v^{2}}{2} .
\end{gathered}
$$

When plotting the constitutive law $p=m v$, with $p$ in the $\mathrm{y}$-axis and $\mathrm{v}$ in the $\mathrm{x}$-axis, the area underneath the line represents the energy and the area above the line the coenergy. Notice that the kinetic coenergy is often called kinetic energy in most literatures. The term coenergy is omitted. The reason that happens is that for linear systems the energy equals the coenergy, making the introduction of a new concept unnecessary. For relativistic situations, the constitutive law changes. Now, a nonlinear relation is analysed. Energy and coenergy now differ. The linear equation for the momentum $p$ used to give the problem similar areas and consequently similar energies. The relativistic nonlinear equation gives rise to different areas and different quantities. Finally, we define energy functions for electrical systems as

Energy:

$$
\begin{aligned}
\mathcal{T}_{m} & =\mathcal{T}_{m}(\lambda) \\
\mathcal{W}_{e} & =\mathcal{W}_{e}(q)
\end{aligned}
$$

Coenergy:

$$
\begin{gathered}
\mathcal{W}_{m}=\mathcal{W}_{m}(\dot{q}), \\
\mathcal{V}_{e}=\mathcal{V}_{e}(v) .
\end{gathered}
$$


The magnetic energy $\mathcal{T}_{m}$ is described as a fuction of the flux linkage $\lambda . \mathcal{W}_{m}$ is the magnetic coenergy described as a function of the current $\dot{q}$. The electric coenergy $\mathcal{V}_{e}$ varies with the voltage $v$. The electric energy $\mathcal{W}_{e}$ varies with the charge $q$. Although their difference in the choice of independent variables, energy functions $\mathcal{T}_{m}$ and $\mathcal{W}_{m}$, as well as $\mathcal{V}_{e}$ and $\mathcal{W}_{e}$ have the same units. Note that the magnetic energy and coenergy are functions that can be related through a constitutive law and it depends on the characteristics of the system, just like the example of the moving particle in the mechanical domain. The same holds true to electric energy and coenergy functions. Again, it is important to emphasize that these functions, for magnetic and electric parts separately, may not always be the same, the equality is a characteristic of linear systems only. A benefit of the coenergy is the advantage to calculate mechanical torques and forces of magnetic or electrical origin. It is also interesting to analyze the relation between energies with a graphical approach.

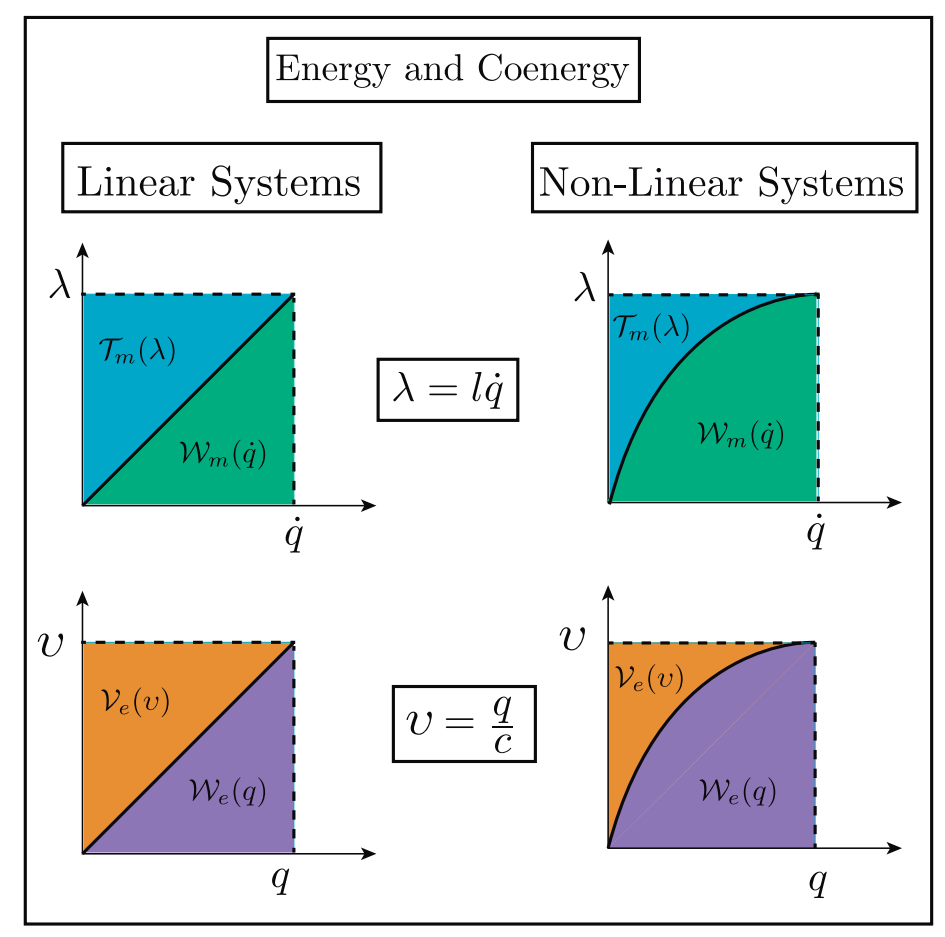

Figure 6: Graphics of energy and coenergy for linear and non-linear systems .

These functions are related through theirs sum, which equals the sum of the multiplication of two independent variables, which are the chosen possibilities to describe electrical systems: $q$ and its derivative $\dot{q}$ and $\lambda_{f}$ and its derivative $e_{f}$. To verify this information an analysis of the graphics is sufficient. For $\mathrm{N}$ electrical terminals:

$$
\begin{aligned}
& \mathcal{T}_{m}+\mathcal{W}_{m}=\sum_{i=1}^{N}\left(\lambda_{f}\right)_{i} \dot{q}_{i} \\
& \mathcal{V}_{e}+\mathcal{W}_{e}=\sum_{i=1}^{N}\left(e_{f}\right)_{i} q_{i}
\end{aligned}
$$




\section{Modeling Technique for Electromechanical Systems}

\subsection{Electromechanical Coupling}

One of the most important subjects when electromechanical systems comes to mind is the electromechanical coupling [19] and how it should appear in the equations of the system. By a sum of energy functions of the electrical part (they are presented in section 3.2 ), it is possible to obtain the electromechanical coupling of the system. The energy being discussed in these functions is the energy between coupling terminals. A coupling terminal can store magnetic or electrical energy, or both.

First, it is shown how to obtain the electromechanical coupling of a system which stores magnetic energy, then the same method is applied to a system with electrical energy stored. To simplify calculus, it is supposed that the system has only one coupled terminal:

$$
\begin{gathered}
\mathcal{T}_{m}+\mathcal{W}_{m}=\dot{q} \lambda \\
\frac{d}{d t}\left(\mathcal{T}_{m}+\mathcal{W}_{m}\right)=\frac{d}{d t}(\dot{q} \lambda), \\
\frac{d}{d t} \mathcal{T}_{m}+\frac{d}{d t} \mathcal{W}_{m}=\frac{d}{d t}(\dot{q} \lambda), \\
\left(\dot{q} \frac{d \lambda}{d t}-f_{e} \frac{d x}{d t}-\tau_{e} \frac{d \alpha}{d t}\right)+\left(\frac{d \mathcal{W}_{m}}{d \dot{q}} \frac{d \dot{q}}{d t}+\frac{d \mathcal{W}_{m}}{d x} \frac{d x}{d t}+\frac{d \mathcal{W}_{m}}{d \alpha} \frac{d \alpha}{d t}\right)=\left(\dot{q} \frac{d \lambda}{d t}+\lambda \frac{d \dot{q}}{d t}\right), \\
\left(\frac{d \mathcal{W}_{m}}{d \dot{q}}-\lambda\right) \frac{d \dot{q}}{d t}+\left(\frac{d \mathcal{W}_{m}}{d x}-f_{e}\right) \frac{d x}{d t}+\left(\frac{d \mathcal{W}_{m}}{d \alpha}-\tau_{e}\right) \frac{d \alpha}{d t}=0
\end{gathered}
$$

Now, the same is done for the electric part:

$$
\begin{gathered}
\mathcal{V}_{e}+\mathcal{W}_{e}=v q \\
\frac{d}{d t}\left(\mathcal{V}_{e}+\mathcal{W}_{e}\right)=\frac{d}{d t}(v q), \\
\frac{d}{d t} \mathcal{V}_{e}+\frac{d}{d t} \mathcal{W}_{e}=\frac{d}{d t}(v q) \\
\left(v \frac{d q}{d t}-f_{e} \frac{d x}{d t}-\tau_{e} \frac{d \alpha}{d t}\right)+\left(\frac{d \mathcal{W}_{e}}{d v} \frac{d v}{d t}+\frac{d \mathcal{W}_{e}}{d x} \frac{d x}{d t}+\frac{d \mathcal{W}_{e}}{d \alpha} \frac{d \alpha}{d t}\right)=\left(v \frac{d q}{d t}+q \frac{d v}{d t}\right), \\
\left(\frac{d \mathcal{W}_{e}}{d v}-q\right) \frac{d v}{d t}+\left(\frac{d \mathcal{W}_{e}}{d x}-f_{e}\right) \frac{d x}{d t}+\left(\frac{d \mathcal{W}_{e}}{d \alpha}-\tau_{e}\right) \frac{d \alpha}{d t}=0 .
\end{gathered}
$$


It is possible to satisfy the equations as $d v, d x, d \dot{q}$ and $d \alpha$ can have arbitrary values. By requiring the coefficients to be zero one gets

- $\frac{d \mathcal{W}_{e}}{d v}=q$

- $\frac{d \mathcal{W}_{e}}{d x}=f_{e}$

- $\frac{d \mathcal{W}_{e}}{d \alpha}=\tau_{e}$

- $\frac{d \mathcal{W}_{m}}{d \dot{q}}=\lambda$

- $\frac{d \mathcal{W}_{m}}{d x}=f_{e}$,

- $\frac{d \mathcal{W}_{m}}{d \alpha}=\tau_{e}$

Depending on the characteristics of the system, the coupling can be obtained by the equations above and if the stored energy is known, the electrical and mechanical terminal relations can now be calculated. To differ from the magnetic coenergy and the electric energy, $\mathcal{W}_{m}$ and $\mathcal{W}_{e}$, we represent the coupling term as $\mathcal{W}^{*}$.

\subsection{Lagrangians for Electromechanical Systems}

By simply grouping the mechanical kinetic coenergy $T$ and potential energy $V$ with the magnetic coenergy $W_{m}$ and electrical energy $W_{e}$ in the Lagrange's equations, a formulation for electromechanical system can be developed [6]. For a generalized coordinate $z$ one gets

$$
\begin{gathered}
\frac{d}{d t}\left(\frac{\partial}{\partial \dot{z}_{i}}\left(T+\mathcal{W}_{m}\right)\right)-\frac{\partial}{\partial z_{i}}\left(T+\mathcal{W}_{m}\right)+\frac{\partial}{\partial z_{i}}\left(V+\mathcal{W}_{e}\right)=0, \\
\frac{d}{d t}\left(\frac{\partial}{\partial \dot{z}_{i}}\left(T+\mathcal{W}_{m}\right)\right)-\frac{\partial}{\partial z_{i}}\left(T+\mathcal{W}_{m}-V-\mathcal{W}_{e}\right)=0 .
\end{gathered}
$$

Introducing the Lagrangian $\mathcal{L}$ for electromechanical systems

$$
\mathcal{L}=T-V+\mathcal{W}_{m}-\mathcal{W}_{e},
$$

where the Lagrangian for mechanical systems $L$ and electrical systems $\mathcal{W}$ are defined by:

$$
\begin{gathered}
L=T-V, \\
\mathcal{W}=\mathcal{W}_{m}-\mathcal{W}_{e} .
\end{gathered}
$$


As a result an extended Lagrangian is obtained:

$$
\mathcal{L}=L+\mathcal{W}
$$

By substituting in equation 55 we get:

$$
\frac{d}{d t}\left(\frac{\partial \mathcal{L}}{\partial \dot{z}_{i}}\right)-\frac{\partial \mathcal{L}}{\partial z_{i}}=0 .
$$

Now, a generalized function $Q$ must be added to the right side of the equation 60 . A clever way to represent this generalized function is with an index $i$. For example, a $Q_{1}$ could represent the imposed forces and the dissipation of the mechanical part and a $Q_{2}$, the imposed voltages and the dissipation of the electrical part. We define

$$
Q_{i}=F_{i}-\frac{\partial D_{i}}{\partial \dot{z}_{i}}
$$

The dynamical equations for electromechanical systems are obtained from

$$
\begin{gathered}
\frac{d}{d t}\left(\frac{\partial \mathcal{L}}{\partial \dot{z}_{i}}\right)-\frac{\partial \mathcal{L}}{\partial z_{i}}=Q_{i} \\
\frac{d}{d t}\left(\frac{\partial \mathcal{L}}{\partial \dot{z}_{i}}\right)-\frac{\partial \mathcal{L}}{\partial z_{i}}=F_{i}-\frac{\partial D_{i}}{\partial \dot{z}_{i}} .
\end{gathered}
$$

\subsection{Step by Step: Lagrangians for Electromechanical Systems}

\section{[Step 1: Identifying the problem]}

First step consists in identify how many mechanical and electrical variables the system needs to be described. In this project the electrical part is described by the coordinate $q$ (for electric charges) and the mechanical part is described by the coordinates $x$ (for translational displacements) and $\alpha, \phi$, or $\theta$ (for angular displacements).

\section{[Step 2: Electromechanical Coupling]}

Step 2 consists in determining the electromechanical coupling of the system. Electromechanical couplings are not always evident. An identification of which terminals demonstrate interactions between subsystems seems to be a good start. After finding the coupling terminals, some other questions might need answers. It is important to know from what kind of energy the coupling is originated and what equation to use. 
Couplings of electric origin can be identified by the equations

$$
\begin{aligned}
& \frac{d \mathcal{W}_{e}^{*}}{d v}=q, \\
& \frac{d \mathcal{W}_{e}^{*}}{d x}=f_{e}, \\
& \frac{d \mathcal{W}_{e}^{*}}{d \alpha}=\tau_{e} .
\end{aligned}
$$

Couplings of magnetic origin can be identified by the equations:

$$
\begin{aligned}
& \frac{d \mathcal{W}_{m}^{*}}{d \dot{q}}=\lambda, \\
& \frac{d \mathcal{W}_{m}^{*}}{d x}=f_{e}, \\
& \frac{d \mathcal{W}_{m}^{*}}{d \alpha}=\tau_{e} .
\end{aligned}
$$

After the coupling is obtained, it must be inserted in $\mathcal{W}$. The sign of the coupling will depend of its origin. If it is originated from a magnetic coupling field, the sign should be positive. If it is originated from an electric coupling field, the sign should be negative. The coupling must be added to its respective energy, namely

$$
\begin{gathered}
\mathcal{W}=\left(\mathcal{W}_{m}+\mathcal{W}^{*}\right)-\mathcal{W}_{e} \quad \text { (For a magnetic coupling) } \\
\mathcal{W}=\mathcal{W}_{m}-\left(\mathcal{W}_{e}+\mathcal{W}^{*}\right) \quad \text { (For an electrical coupling) }
\end{gathered}
$$

\section{[Step 3: Kinetic Coenergy and Potential Energy]}

Describe kinetic coenergy and potential energy of the mechanical part.

Translational and rotational kinetic coenergy are represented by $T$.

Gravitational and elastic potential energy are represented by $V$.

\section{[Step 4: Magnetic Coenergy and Electric Energy]}

Describe magnetic coenergy and electrical energy of the non-coupled electrical part.

Magnetic coenergy is represented by $\mathcal{W}_{m}$.

Electrical potential energy is represented by $\mathcal{W}_{e}$. 
[Step 5: Lagrangian for Electromechanical Systems]

We write the Lagrangian:

$$
\begin{gathered}
\mathcal{L}=L+\mathcal{W} \\
\mathcal{L}=T-V+\mathcal{W}_{m}-\mathcal{W}_{e} \pm \mathcal{W}^{*} .
\end{gathered}
$$

\section{[Step 6: Generalized Forces]}

We introduce the function $Q$.

Where $D$ is a dissipative function for resistances and viscous frictions. $F$ includes imposed forces and voltages. For each degree of freedom $i$, the $Q$ function takes the form:

$$
Q_{i}=F_{i}-\frac{\partial D_{i}}{\partial \dot{z}_{i}}
$$

\section{[Step 7: Lagrange's Equations]}

Being $z_{i}$ a generalized coordinate:

$$
\frac{d}{d t}\left(\frac{\partial \mathcal{L}}{\partial \dot{z}_{i}}\right)-\frac{\partial \mathcal{L}}{\partial z_{i}}=F_{i}-\frac{\partial D_{i}}{\partial \dot{z}_{i}}
$$




\section{Dynamics of Electromechanical Systems}

\subsection{Scotch-Yoke Mechanism}

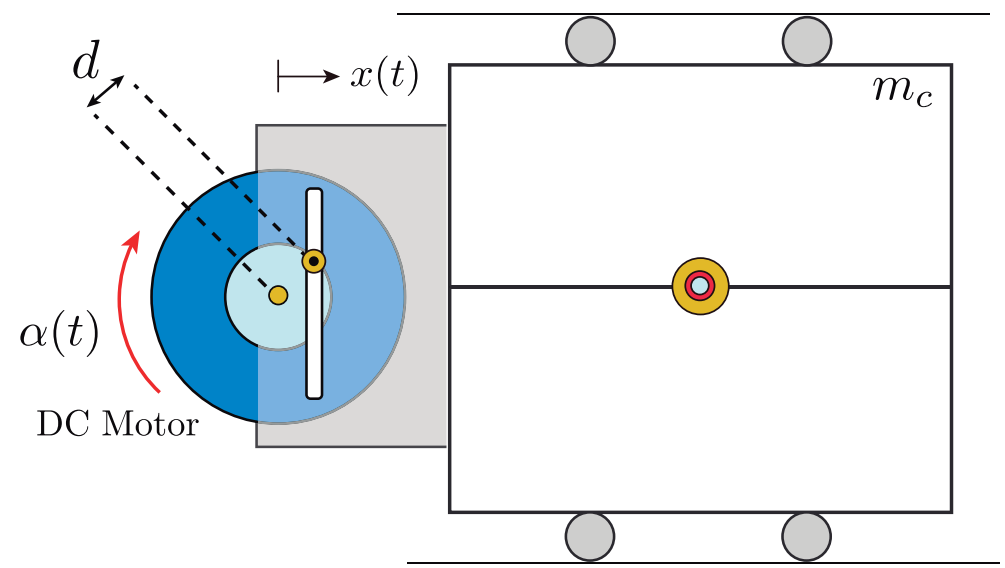

Figure 7: Cart-motor system with a scotch-yoke mechanism.

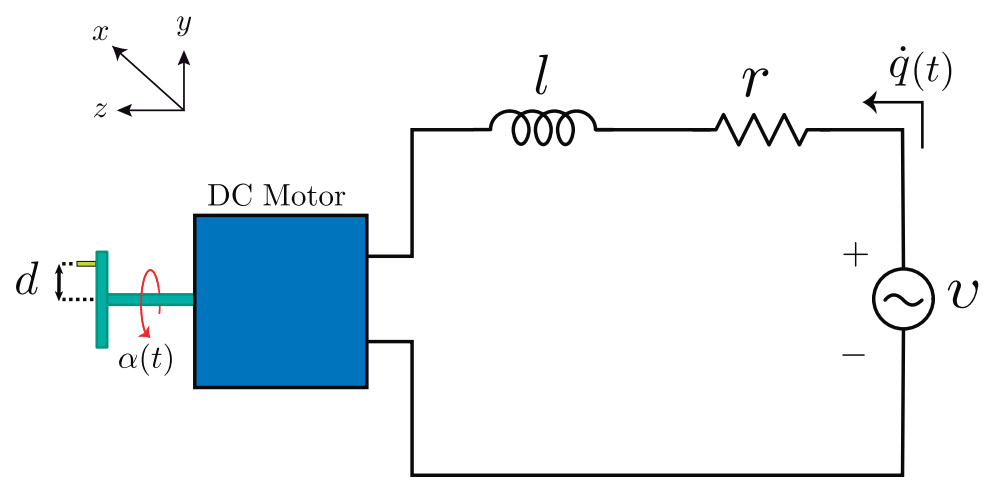

Figure 8: DC motor. 
As previously stated, the first electromechanical system analysed in this project is composed by a cart driven by a DC motor coupled by a mechanism called scotch-yoke. This system has been an object of study to analyze the behavior of electromechanical systems, see[16, 14]. Where $v$ is the voltage source, $\dot{q}$ is the electric current, $\alpha$ is the angular displacement of the disk, $l$ is the electric inductance and $r$ is the electric resistance. The motor is coupled to the cart through a pin that slides into a slot machined in a plate that is attached to the cart, $d$ is the distance related to the eccentricity of the pin. In this system, there are two coupling mechanisms. One of them is the electromechanical coupling. It exists when a disk is attached to a motor. The other is a mechanical coupling. It exists when a mechanical part is coupled to another mechanical part. In this case, the scotch-yoke mechanism is transforming the rotational movement of the disk in a translational movement of the cart.

\section{[Step 1: Identifying the problem]}

This system has three movements involved. The rotational movement of the disk, the translational movement of the cart and the moving charges in the electrical part. It has one constraint due to the scotch-yoke mechanism. It has two degrees of freedom. The electrical part is described by the charge $q$ and the mechanical part by the angular displacement $\alpha$.

\section{[Step 2: Electromechanical coupling]}

For the electromechanical coupling, as it stores magnetic energy in the coupling terminal, it is interesting to analyse the equation

$$
\frac{d \mathcal{W}_{m}^{*}}{d \alpha}=\tau_{e}
$$

In most DC motors, the torque $\tau_{e}$ is proportional to the armature current $\dot{q}$ and the strength of the magnetic field denoted by $k_{e}$, which is the motor electromagnetic force constant $[12]$.

$$
\tau_{e}=k_{e} \dot{q}
$$

Substituting equation 77 in 76 gives

$$
\begin{gathered}
\frac{d \mathcal{W}_{m}^{*}}{d \alpha}=k_{e} \dot{q}, \\
d \mathcal{W}_{m}^{*}=k_{e} \dot{q} d \alpha .
\end{gathered}
$$

Now, the magnetic energy stored in the terminals of the coupling equals the electromechanical coupling of the system. For $\mathcal{W}_{m}^{*}=\mathcal{W}^{*}$ :

$$
\mathcal{W}^{*}=k_{e} \dot{q} \alpha
$$


[Step 3: Kinetic coenergy and potential energy]

$$
T=\frac{j_{m} \dot{\alpha}^{2}}{2}+\frac{m_{c} \dot{x}^{2}}{2}
$$

Where $j_{m}$ is the inertia moment of the motor. Besides the electromechanical coupling, there is another one. The coupling between the motor and the cart is made by a mechanism called scotch yoke, which transforms the rotational movement of the motor in the translation motion of the cart, leading to the relations

$$
\begin{gathered}
x=d \cos (\alpha), \\
\dot{x}=-d \sin (\alpha) \dot{\alpha},
\end{gathered}
$$

Substituting 83 in 81 gives

$$
T=\frac{j_{m} \dot{\alpha}^{2}}{2}+\frac{m_{c}[-d \sin (\alpha) \dot{\alpha}]^{2}}{2} .
$$

This system has no potential energy for the mechanical part, i. e.

$$
V=0 \text {. }
$$

[Step 4: Magnetic coenergy and electric energy]

For the electrical subsystem we write the magnetic coenergy:

$$
\mathcal{W}_{m}=\frac{l \dot{q}^{2}}{2}+\mathcal{W}^{*}
$$

Substituting 80 in 86 leads to

$$
\mathcal{W}_{m}=\frac{l \dot{q}^{2}}{2}+k_{e} \dot{q} \alpha
$$

This system has no electrical potential energy.

$$
\mathcal{W}_{e}=0
$$

[Step 5: Lagrangian for electromechanical systems]

$$
\begin{gathered}
\mathcal{L}=L+\mathcal{W}, \\
\mathcal{L}=T-V+\mathcal{W}_{m}-\mathcal{W}_{e} \\
\mathcal{L}=\frac{j_{m} \dot{\alpha}^{2}}{2}+\frac{m_{c}[-d \sin (\alpha) \dot{\alpha}]^{2}}{2}+\frac{l \dot{q}^{2}}{2}+k_{e} \dot{q} \alpha .
\end{gathered}
$$




\section{[Step 6: Generalized forces]}

For the mechanical coordinate $\alpha$ :

$$
\begin{gathered}
F_{1}=0, \\
D_{1}=\frac{b_{m} \dot{\alpha}^{2}}{2}, \\
Q_{1}=F_{1}-\frac{\partial D_{1}}{\partial \dot{\alpha}}, \\
Q_{1}=-b_{m} \dot{\alpha} .
\end{gathered}
$$

For the electrical coordinate $q$ :

$$
\begin{gathered}
F_{2}=v, \\
D_{2}=\frac{r \dot{q}^{2}}{2}, \\
Q_{2}=F_{2}-\frac{\partial D_{2}}{\partial \dot{q}}, \\
Q_{2}=v-r \dot{q} .
\end{gathered}
$$

[Step 7: Lagrange's equations]

$$
\begin{aligned}
& \frac{d}{d t}\left(\frac{\partial \mathcal{L}}{\partial \dot{\alpha}}\right)-\frac{\partial \mathcal{L}}{\partial \alpha}=Q_{1}, \\
& \frac{d}{d t}\left(\frac{\partial \mathcal{L}}{\partial \dot{q}}\right)-\frac{\partial \mathcal{L}}{\partial q}=Q_{2} .
\end{aligned}
$$

For equations 100 and 101, respectively:

$$
\begin{gathered}
\ddot{\alpha}\left[j_{m}+m_{c} d^{2} \sin ^{2}(\alpha)\right]+\dot{\alpha}\left[b_{m}+m_{c} d^{2} \sin (\alpha) \cos (\alpha) \dot{\alpha}\right]-k_{e} \dot{q}=0, \\
l \ddot{q}+r \dot{q}+k_{e} \dot{\alpha}=v,
\end{gathered}
$$




\subsection{Crank-Slider Mechanism}

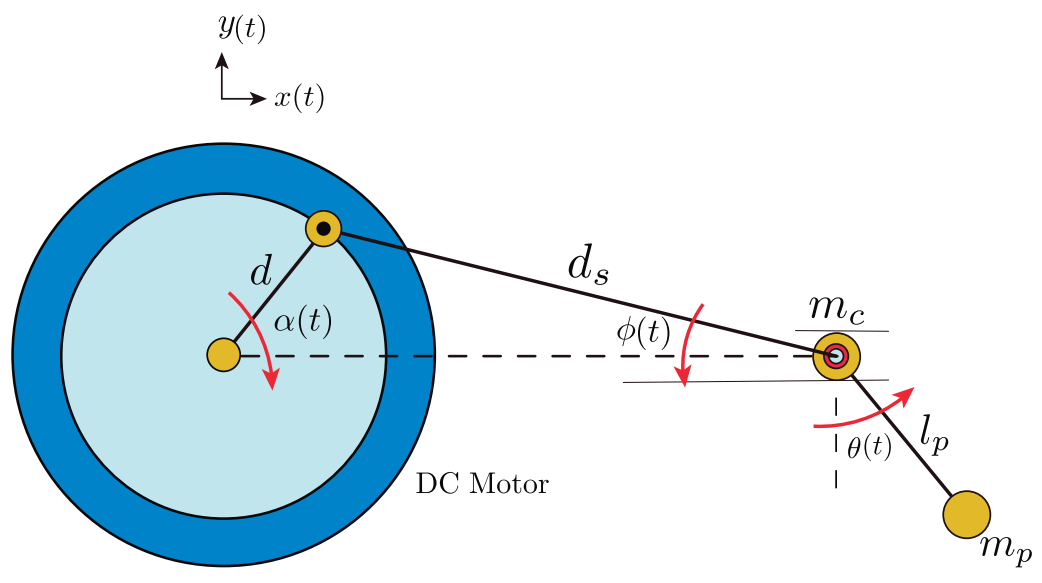

Figure 9: Electromechanical system with a crank-slider mechanism.

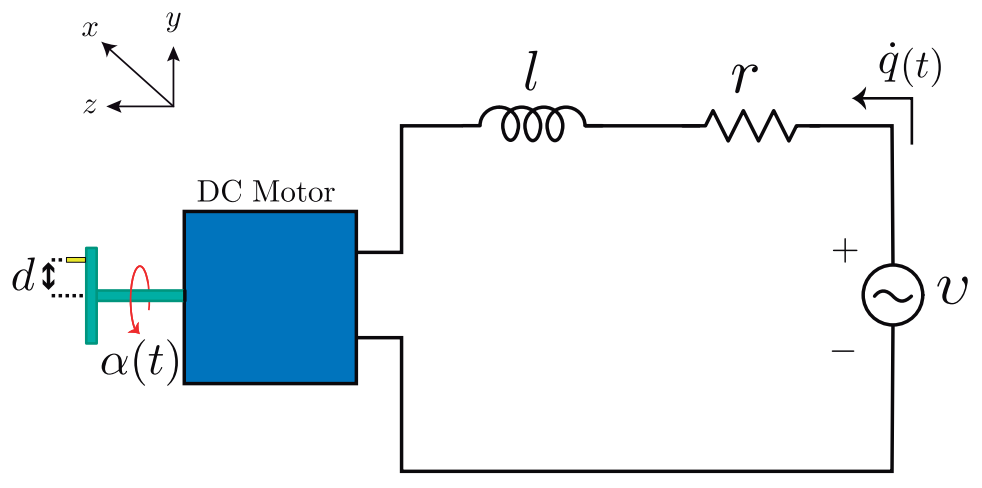

Figure 10: DC motor.

The second system analysed in this project consists of a crank-slider mechanism linked to a pendulum of mass $m_{p}$ and length $l_{p}$. There is not much difference to the previous system when it comes to the electrical subsystem. The coordinates of the electrical subsystem are the same. The particle with no mass in the mechanical part substitutes the cart. This system has also a DC motor. There are viscous frictions in the disk coupled to the motor $\left(b_{m}\right)$, in the displacement of the particle with no mass $\left(b_{c}\right)$ and in the movement of the pendulum $\left(b_{p}\right)$. It differs from the first in the choice of coupling mechanism. The distance related to the eccentricity of the crank is $d$. The length of the shaft is $d_{s}$. The angles in the crank and in the shaft are given by $\alpha(t)$ and $\phi(t)$, respectively. The angular displacement of the pendulum is given by $\theta(t)$.

\section{[Step 1: Identifying the problem]}

This system has four movements involved. The rotational movement of the disk, the rotational movement of the shaft, the rotational movement of the pendulum and the moving charges in the electrical part. It has one constraint due to the crank-slider mechanism. It has three degrees of freedom. The electrical part is described by the charge $q(t)$ and 
the mechanical part by the angular displacements $\alpha(t)$ and $\theta(t)$.

\section{[Step 2: Electromechanical coupling]}

Comparing to the first system analysed, the first coupling do not change:

$$
\mathcal{W}^{*}=k_{e} \dot{q} \alpha .
$$

\section{[Step 3: Kinetic coenergy and potential energy]}

For the kinetic coenergy and the potential energy of the mechanical part:

$$
T=\frac{j_{m} \dot{\alpha}^{2}}{2}+\frac{m_{p}\left(\dot{x}^{2}+\dot{y}^{2}\right)}{2},
$$

besides the electromechanical coupling, there is another one made by the crank-slider mechanism. First, we begin by writing the position of the pendulum:

$$
\begin{gathered}
x=d \cos (\alpha)+d_{s} \cos (\phi)+l_{p} \sin (\theta), \\
y=-l_{p} \cos (\theta) .
\end{gathered}
$$

The angles $\alpha$ and $\phi$ are related by

$$
\cos (\phi)=\left(1-\frac{d^{2}}{d_{s}^{2}} \sin (\alpha)^{2}\right)^{\frac{1}{2}} .
$$

Substituting in the equation and deriving in time gives

$$
\begin{gathered}
\dot{x}=-d \dot{\alpha}\left(\sin (\alpha)+\frac{d \sin (\alpha) \cos (\alpha)}{d_{s}\left(1-\frac{d^{2}}{d_{s}^{2}} \sin (\alpha)^{2}\right)^{\frac{1}{2}}}\right)+l_{p} \dot{\theta} \cos (\theta), \\
\dot{y}=l_{p} \dot{\theta} \sin (\theta),
\end{gathered}
$$

introducing the function $\mathcal{K}(\alpha)$ :

$$
\mathcal{K}(\alpha)=\sin (\alpha)+\frac{d \sin (\alpha) \cos (\alpha)}{d_{s}\left(1-\frac{d^{2}}{d_{s}^{2}} \sin (\alpha)^{2}\right)^{\frac{1}{2}}}
$$

As a result, it is possible to simplify $\dot{x}$. For the horizontal and vertical velocities of the pendulum:

$$
\dot{x}=-d \dot{\alpha} \mathcal{K}+l_{p} \dot{\theta} \cos (\theta),
$$




$$
\dot{y}=l_{p} \dot{\theta} \sin (\theta) .
$$

Rewriting the kinetic coenergy:

$$
T=\frac{j_{m} \dot{\alpha}^{2}}{2}+\frac{m_{p}}{2}\left[\left(l_{p} \dot{\theta} \cos (\theta)-d \dot{\alpha} \mathcal{K}\right)^{2}+\left(l_{p} \dot{\theta} \sin (\theta)\right)^{2}\right] .
$$

For the potential energy of the system we write the gravitational potential energy:

$$
V=-m_{p} g l_{p} \cos (\theta)
$$

[Step 4: Magnetic coenergy and electric energy]

For the magnetic coenergy of the system:

$$
\begin{gathered}
\mathcal{W}_{m}=\frac{l \dot{q}^{2}}{2}+\mathcal{W}^{*} \\
\mathcal{W}_{m}=\frac{l \dot{q}^{2}}{2}+k_{e} \dot{q} \alpha .
\end{gathered}
$$

For the electric potential energy of the system:

$$
\mathcal{W}_{e}=0
$$

[Step 5: Lagrangian for electromechanical systems]

$$
\begin{gathered}
\mathcal{L}=L+\mathcal{W}, \\
\mathcal{L}=T-V+\mathcal{W}_{m}-\mathcal{W}_{e}, \\
\mathcal{L}=\frac{j_{m} \dot{\alpha}^{2}}{2}+\frac{m_{p}}{2}\left[\left(l_{p} \dot{\theta} \cos (\theta)-d \dot{\alpha} \mathcal{K}\right)^{2}+\left(l_{p} \dot{\theta} \sin (\theta)\right)^{2}\right]+m_{p} g l_{p} \cos (\theta)+\frac{l \dot{q}^{2}}{2}+k_{e} \dot{q} \alpha .
\end{gathered}
$$

\section{[Step 6: Generalized forces]}

For the mechanical coordinate $\alpha$ :

$$
\begin{gathered}
F_{1}=0 \\
D_{1}=\frac{b_{c}(-d \dot{\alpha} \mathcal{K})^{2}}{2}+\frac{b_{m} \dot{\alpha}^{2}}{2} \\
Q_{1}=F_{1}-\frac{\partial D_{1}}{\partial \dot{\alpha}}
\end{gathered}
$$




$$
Q_{1}=-b_{c} d^{2} \mathcal{K}^{2} \dot{\alpha}-b_{m} \dot{\alpha}
$$

For the mechanical coordinate $\theta$ :

$$
\begin{gathered}
F_{2}=0 \\
D_{2}=\frac{b_{p}\left(\left(l_{p} \dot{\theta} \sin (\theta)\right)^{2}+\left(l_{p} \dot{\theta} \sin (\theta)\right)^{2}\right)}{2} \\
Q_{2}=F_{2}-\frac{\partial D_{2}}{\partial \dot{\theta}} \\
Q_{2}=-b_{p} l_{p}^{2} \dot{\theta}
\end{gathered}
$$

For the electrical coordinate $q$ :

$$
\begin{gathered}
F_{3}=v, \\
D_{3}=\frac{r \dot{q}^{2}}{2}, \\
Q_{3}=F_{3}-\frac{\partial D_{3}}{\partial \dot{q}}, \\
Q_{3}=v-r \dot{q} .
\end{gathered}
$$

[Step 7: Lagrange's equations]

$$
\begin{aligned}
& \frac{d}{d t}\left(\frac{\partial \mathcal{L}}{\partial \dot{\alpha}}\right)-\frac{\partial \mathcal{L}}{\partial \alpha}=Q_{1}, \\
& \frac{d}{d t}\left(\frac{\partial \mathcal{L}}{\partial \dot{\theta}}\right)-\frac{\partial \mathcal{L}}{\partial \theta}=Q_{2}, \\
& \frac{d}{d t}\left(\frac{\partial \mathcal{L}}{\partial \dot{q}}\right)-\frac{\partial \mathcal{L}}{\partial q}=Q_{3} .
\end{aligned}
$$

For equations 134, 135 and 136, respectively:

$$
\begin{aligned}
\ddot{\alpha}\left[j_{m}+m_{p} d^{2} \mathcal{K}^{2}\right]+\dot{\alpha}\left[b_{m}+b_{c} d^{2} \mathcal{K}^{2}+m_{p} d^{2} \mathcal{K} \dot{\mathcal{K}}\right]+\ddot{\theta}\left[-m_{p} l_{p} d \cos (\theta) \mathcal{K}\right]+ \\
\dot{\theta}\left[m_{p} l_{p} d \sin (\theta) \dot{\theta} \mathcal{K}\right]-k_{e} q=0,
\end{aligned}
$$




$$
\begin{gathered}
\ddot{\theta}\left[m_{p} l_{p}^{2}\right]+\dot{\theta}\left[b_{p} l_{p}^{2}\right]+\ddot{\alpha}\left[-m_{p} l_{p} d \cos (\theta) \dot{\alpha} \mathcal{K} \dot{\mathcal{K}}\right]+\dot{\alpha}\left[-m_{p} l_{p} d \cos (\theta) \dot{\mathcal{K}}\right]+m_{p} l_{p} g \sin (\theta)=0, \\
l \ddot{q}+r \dot{q}+k_{e} \dot{\alpha}=v .
\end{gathered}
$$

\section{Conclusions}

The objective of this project was to formulate a Lagrangian for electromechanical systems. It is not possible to deal with electromechanical systems without knowing the electrical part, please see [15]. Some incorrect methods consist in mimicking the standard derivations of purely mechanical systems to electromechanical systems . There are many articles, thesis and books that say they are able to find the equations of electromechanical systems through a Lagrangian. Frequently, they solve problems only for the mechanical part or make mistakes in the formulation that leads to the correct solution, see $[1,2,3,4,5,8]$. For more details about the errors, please see [17].

When systems composed by different parts are analysed, the term that makes the connection between the two must be discussed. The electromechanical coupling is an essential tool to formulate a Lagrangian. To introduce the electromechanical coupling, it was necessary to present the difference between energy and coenergy and how energy is transferred from one place to another in an electromechanical model.

In future works some developments may be followed: How to formulate a Lagrangian for a three dimensional space ploblem and the possibility of formulating a Lagrangian for another combination of physical systems such as mechanical with fluid or thermo.

\section{References}

[1] Rafael Avanço, Angelo Tusset, Marcelo Suetake, Helio Navarro, José Balthazar, and Airton Nabarrete, The pendulum dynamic analysis with dc motors and generators for sea waves energy harvest, 122017.

[2] Rafael Henrique Avanço, Análise da dinâmica não-linear de pêndulos com excitação paramétrica por um mecanismo biela-manivela, Doctor thesis, USP-São Carlos (2015).

[3] Rafael Henrique Avanço, Angelo Marcelo Tusset, José Manoel Balthazar, Airton Nabarrete, and Helio Aparecido Navarro, On nonlinear dynamics behavior of an electro-mechanical pendulum excited by a nonideal motor and a chaos control taking into account parametric errors, Journal of the Brazilian Society of Mechanical Sciences and Engineering 40 (2018), 23.

[4] D Belato, Análise não linear de sistemas dinâmicos holonômos não ideais, Tese de doutorado, Doctor thesis, Unicamp, 2002. 
[5] Debora Belato, Hans I Weber, José Manoel Balthazar, and Dean T Mook, Chaotic vibrations of a nonideal electro-mechanical system, International Journal of Solids and Structures 38 (2001), no. 10-13, 1699-1706.

[6] Robert H Bishop, Mechatronic systems sensors and actuators-fundamentals and modelling, the mechatronics handbook, Boca Raton, FL: CRC Press Inc, 2008.

[7] Herbert B Callen, Thermodynamics and an introduction to thermostatistics, 1998.

[8] Livija Cveticanin, Miodrag Zukovic, and Jose Manoel Balthazar, Dynamics of mechanical systems with non-ideal excitation, Springer, 2018.

[9] Márcio José Horta Dantas, Rubens Sampaio, and Roberta Lima, Existence and asymptotic stability of periodic orbits for a class of electromechanical systems: a perturbation theory approach, Zeitschrift für angewandte Mathematik und Physik 67 (2016), no. 1, 2 .

[10] MJH Dantas, R Sampaio, and R Lima, Asymptotically stable periodic orbits of a coupled electromechanical system, Nonlinear Dynamics 78 (2014), no. 1, 29-35.

[11] Werner Haas, Kurt Schlacher, and R Gahleitener, Modeling of electromechanical systems, Johannes Kepler University Linz, Department of Automatic Control and Control Systems Technology, Preliminary Version 13 (2000).

[12] Austin Hughes and William Drury, Electric motors and drives: fundamentals, types and applications, Newnes, 2013.

[13] Dimitri Jeltsema and Jacquelien MA Scherpen, Multidomain modeling of nonlinear networks and systems, IEEE Control Systems 29 (2009), no. 4.

[14] R Lima and R Sampaio, Stochastic analysis of an electromechanical coupled system with embarked mass, Mecânica Computacional 31 (2012), 2709-2733.

[15] R. Lima and R. Sampaio, Pitfalls in the dynamics of coupled electromechanical systems, Tech. report, PUC-Rio, 042018.

[16] Roberta Lima and Rubens Sampaio, Two parametric excited nonlinear systems due to electromechanical coupling, Journal of the Brazilian Society of Mechanical Sciences and Engineering 38 (2016), no. 3, 931-943.

[17] William Manhães, Rubens Sampaio, Roberta Lima, Peter Hagedorn, and JeanFrançois Deü, Lagrangians for electromechanical systems, (2018).

[18] Dare A Wells, Schaum's outline of theory and problems of lagrangian dynamics: with a treatment of euler's equations of motion, hamilton's equations and hamilton's principle, McGraw-Hill, 1967.

[19] Herbert H Woodson and James R Melcher, Electromechanical dynamics, Wiley, 1968. 